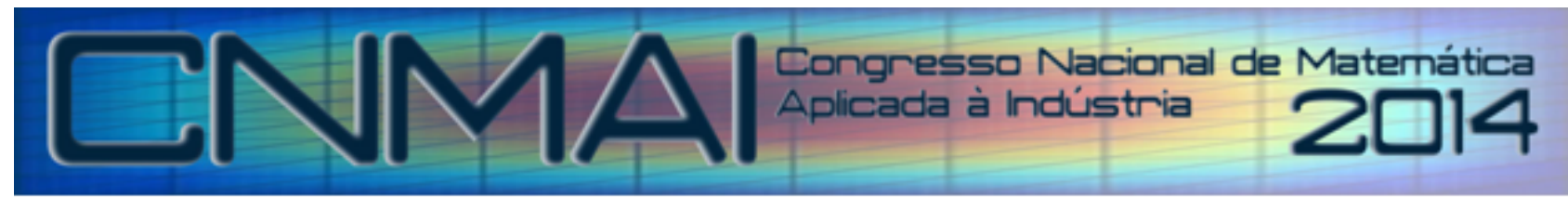

18 a 21 de novembro de 2014, Caldas Novas - Goiás

\title{
ANÁLISE DE ESTABILIDADE E PROJETO DE CONTROLE PELO MÉTODO SDRE PARA UM SISTEMA MAGLEV SIMPLIFICADO
}

\author{
Thalles Denner Ferreira Cabral, ctdf. engmecegmail.com ${ }^{1}$ \\ Fábio Roberto Chavarette, fabioch@mat. feis . unesp.br ${ }^{1}$ \\ ${ }^{1}$ UNESP - Universidade Estadual Paulista - Departamento de Engenharia Mecânica \\ Avenida Brasil, 56, 15385-000, Ilha Solteira, SP, Brasil
}

\begin{abstract}
Resumo. Trens Maglev (Transporte de levitação magnética) são sistemas de transporte que podem atingir grandes velocidades com baixo atrito, em comparação aos trens convencionais roda-trilho. Outros fatores que tornam a tecnologia de trens Maglev muito atrativa é que esta pode diminuir o congestionamento do trafego terrestre e adicionalmente reduzir impactos ambientais. Assim, vários engenheiros ao redor do mundo, desenvolvem projetos relacionados a tecnologia de trens com levitação magnética, sendo que alguns destes projetos já estão num estagio relativamente avançado, por exemplo, os sistemas Maglev experimentais da Alemanha e Japão, os quais têm demonstrado que este modo de transporte pode lucrativamente competir com o transporte aéreo. O primeiro sistema de trem que opera completamente sem contato e sem rodas foi desenvolvido pelos alemães, sendo este um sistema de levitação eletromagnética (EML). Neste trabalho, a estabilidade de um sistema com levitação eletromagnética (EML), tal como o Maglev alemão Transrapid foi analisada a partir de um modelo simplificado por um sistema de massa única sobre um trilho rígido e sem a suspensão secundária; sendo as equações de movimento obtidas pela formulação lagrangiana da mecânica clássica. Por causa da instabilidade do sistema de levitação eletromagnética a força magnética provida do eletroímã deve ser ativamente controlada; assim utilizou-se o método SDRE (Equação de Riccati Dependente do Estado) para o projeto do controlador. Para a simulação do sistema de controle o software MATLAB ${ }^{\circledR}$ é utilizado, sendo mostrado o histórico no tempo das variáveis de estado e a trajetória do sistema controlado no espaço de estados.
\end{abstract}

Palavras-chave: controle SDRE, estabilidade, levitação eletromagnética, modelo matemático, simulação computacional

\section{INTRODUÇÃO}

Um dos sistemas de engenharia que atraem a atenção de diversos pesquisadores é a tecnologia de trens com levitação magnética, chamado de Maglev (Transporte de levitação magnética). O interesse em tais sistemas devem-se ao fato de que estes demonstram ser uma importante alternativa aos meios de transporte de massa convencionais, isto porque, a tecnologia empregada nos trens Maglev é tal que o transporte seja rápido, confortável, seguro, e adicionalmente contribua para o descongestionamento do tráfego rodoviário e/ou urbano, o que consequentemente leva à redução de impactos ambientais como, por exemplo, a redução da emissão de poluentes devido à combustão de combustíveis fosseis, assim, os trens Maglev são ambientalmente corretos (Zhao e Thornton, 1992).

O primeiro sistema de trem que opera completamente sem contato e rodas foi desenvolvido pelos alemães, e está implementado na China desde 2004, sendo este um sistema que interliga o Aeroporto Internacional de Pudong à Estação de Metrô de Long Yang, em Shanghai, por meio de duas vias de $30 \mathrm{~km}$. Para completar esse percurso o trem leva em torno de 8 minutos, atingindo a velocidade de $430 \mathrm{~km} / \mathrm{h}$, o mesmo, ainda é capaz de vencer inclinações de aproximadamente $10 \%$, partindo do repouso até a velocidade de $300 \mathrm{~km} / \mathrm{h}$ em $5 \mathrm{~km}$ (Motta, 2011).

Historicamente tentou-se utilizar a força de oposição de ímãs permanentes com mesma polaridade para desenvolver um sistema de transporte sem atrito, e em 1842 o matemático inglês Samuel Earnshaw provou um teorema (que recebeu seu sobrenome), segundo o qual não é possível realizar uma levitação estática de objetos, qualquer que seja a configuração utilizada de ímãs permanentes fixos. Entretanto, a partir do Teorema de Earnshaw, cientistas de todo o mundo estudam possíveis exceções e formas de garantir a estabilidade de sistemas que utilizam o magnetismo para alcançar a levitação (David, 2009). Tais exceções aparecem, por exemplo em: efeitos quânticos, diamagnetismo, campo oscilante, rotação e feedback (realimentação); este último método é o empregado na tecnologia alemã Transrapid, a qual utiliza suspensão 
eletromagnética $(E M L)$ para a levitação do trem. Pelo fato do Maglev Transrapid ser um sistema cujo equilíbrio é instável, um controlador deve ser implementado, a fim de manter uma certa distância (em torno de $10 \mathrm{~mm}$ ) entre os eletroímãs e o trilho, logo o controle da levitação deve ser eficiente de modo a garantir a operação com segurança. Portanto, a partir de um modelo simplificado do Maglev Transrapid, tem-se como objetivo neste trabalho o estudo da estabilidade e consequentemente propor um projeto de controlador para a estabilização da levitação do veículo.

\subsection{Principio de Funcionamento do Maglev Transrapid}

Como visto anteriormente, o Maglev Transrapid utiliza suspensão eletromagnética, logo, a levitação ocorre através de eletroímãs que são posicionados de forma estratégica em um suporte, assim estes eletroímãs exercem uma força de atração nas placas ferromagnéticas instaladas na aba e nas laterais de uma viga do tipo "T". Logo essa configuração permite obter a sustentação e estabilidade lateral do trem (David, 2009). A Figura 1 mostra um esquema deste tipo de levitação.

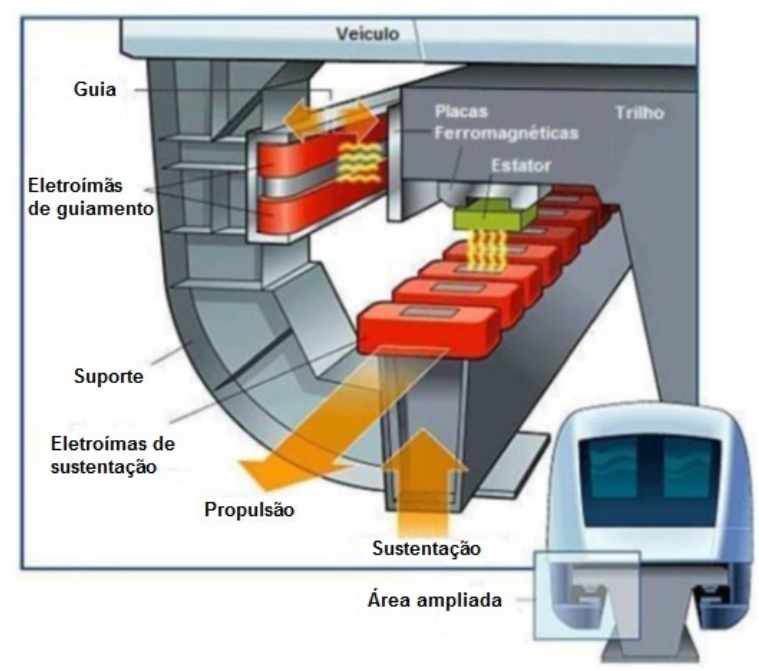

Figura 1. Esquema do sistema de levitação eletromagnética (EML) (Lee et al., 2013).

A propulsão e frenagem são efetuadas por motores síncronos instalados no trilho, sendo estes alimentados por uma corrente alternada de frequência variável. Assim, a velocidade do trem é determinada pela frequência dessa corrente, enquanto que, a aceleração e desaceleração são devido ao sentido do campo girante produzido pelo motor. Caso o sentido do campo seja contrário ao do movimento do trem, a desaceleração ocorre e o motor trabalha como um gerador, e neste caso é possível devolver a energia gerada ao sistema elétrico do trem (Motta, 2011).

\section{MODELO MATEMÁTICO DO SISTEMA MAGLEV SIMPLIFICADO}

Alguns autores como Zhao e Thornton (1992); Shu e Meisinger (2011) propuseram um sistema de massa única sobre um trilho rígido sem a suspensão secundária, como um modelo simplificado, para analisar a estabilidade (considerando apenas a dinâmica vertical) do Maglev alemão Transrapid, e avaliar diferentes projetos de controle. A Figura 2 mostra tal modelo:

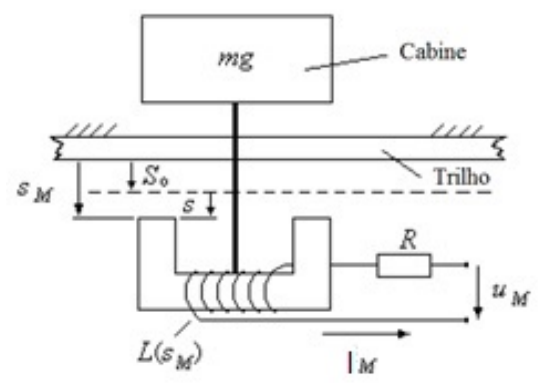

Figura 2. Modelo simplificado para o Maglev Transrapid (Shu e Meisinger, 2011).

onde $m g$ é o peso do veículo (incluindo os eletroímãs); $s_{M}$ é a distância vertical entre o eletroímã e o trilho; $S_{0}$ é a distância vertical entre o trilho e a posição de equilíbrio do veículo; $L\left(s_{M}\right)$ é a indutância da bobina, a qual depende de $s_{M} ; R$ é a resistência da bobina; $I_{M}$ e $u_{M}$ são a corrente e a tensão da bobina respectivamente. 
O fluxo de dispersão e o efeito de correntes parasitas são desprezados neste trabalho. Segundo Shu e Meisinger (2011) sendo a permeabilidade relativa do ferro $\mu_{r} \gg 1$, então, a indutância da bobina $L\left(s_{M}\right)$ pode ser escrita da seguinte maneira:

$$
L\left(s_{M}\right)=\frac{c}{s_{M}} \quad \text { sendo, } \quad c=\frac{\mu_{0} N^{2} A}{2}
$$

onde, $\mu_{0}$ é a constante de indutância; $A$ é a área do polo e $N$ é o número de enrolamentos da bobina.

Seja a seguinte equação de Lagrange:

$$
\frac{d}{d t}\left(\frac{\partial L}{\partial \dot{q}_{k}}\right)-\frac{\partial L}{\partial q_{k}}=Q_{k}^{\prime} \quad \text { sendo, } \quad k=1, \ldots, n
$$

Então, inicialmente define-se a coordenada generalizada como $q_{1}=s_{M}$ e as velocidades generalizadas como $\dot{q}_{1}=$ $\dot{s}_{M}$ e $\dot{q}_{2}=I_{M}$. Assim, a energia cinética $T$ e a energia potencial $U$ do sistema Maglev são respectivamente:

$$
T=\frac{1}{2} m \dot{s}_{M}^{2}+\frac{1}{2} L\left(s_{M}\right) I_{M}^{2} \quad \text { e } \quad U=-m g s_{M}
$$

A lagrangiana do sistema é dada pela diferença entre as duas equações acima, ou seja, $L=T-U$. Logo:

$$
L=\frac{1}{2} m \dot{s}_{M}^{2}+\frac{1}{2} L\left(s_{M}\right) I_{M}^{2}+m g s_{M}
$$

Da equação (2), tem-se que $Q_{k}^{\prime}$ denota a parte das forças generalizadas que não provém de nenhum potencial generalizado (Lemos, 2007). Logo, para o Maglev estudado tem-se o seguinte: $Q_{1}^{\prime}=0$ e $Q_{2}^{\prime}=u_{M}-I_{M} R$

Substituindo (1) em (3) e calculando cada termo de (2) para $k=1 \mathrm{e} k=2$, obtém-se as seguintes equações de movimento do sistema.

$$
\ddot{s}_{M}=-\frac{L\left(s_{M}\right)}{2 s_{M} m} I_{M}^{2}+g \quad \text { e } \quad \dot{I}_{M}=-\frac{R}{L\left(s_{M}\right)} I_{M}+\frac{I_{M}}{s_{M}} \dot{s}_{M}+\frac{1}{L\left(s_{M}\right)} u_{M}
$$

As não linearidades do sistema vêm da indutância não linear devido a geometria do eletroímã e da lei do inverso do quadrado do magnetismo (Zhao e Thornton, 1992).

\subsection{Redução de Ordem do Sistema (Espaço de Estados)}

Substituindo (1) em (4) e definindo as seguintes variáveis de estado $x_{1} \equiv I_{M}, x_{2} \equiv \dot{s}_{M}$ e $x_{3} \equiv s_{M}$, pode-se rescrever o sistema de equações (4) em espaço de estados. Logo:

$$
\begin{aligned}
& \dot{x}_{1}=\frac{x_{3}\left(u_{M}-R x_{1}\right)}{c}+\frac{x_{1} x_{2}}{x_{3}} \\
& \dot{x}_{2}=-\frac{c x_{1}^{2}}{2 m x_{3}^{2}}+g \\
& \dot{x}_{3}=x_{2}
\end{aligned}
$$

\section{ANALISE DE ESTABILIDADE DO PONTO DE EQUILÍBRIO}

Pela definição de ponto de equilíbrio, $\dot{x}_{1}=\dot{x}_{2}=\dot{x}_{3}=0$, quando $x_{1}=x_{1}^{*}, x_{2}=x_{2}^{*}$ e $x_{3}=x_{3}^{*}$, sendo $P=$ $\left(x_{1}^{*}, x_{2}^{*}, x_{3}^{*}\right)$ as coordenadas do ponto de equilíbrio no espaço de estados, ou seja, o ponto no qual o sistema para de se mover no espaço de estados (Monteiro, 2011). Então, quando a força eletromagnética for igual a força gravitacional (peso) do sistema, o mesmo estará num estado de equilíbrio, e assim a velocidade $\dot{s}_{M}$ e aceleração $\ddot{s}_{M}$ (ambas verticais) do Maglev será nula, ou seja $x_{2}^{*}=\dot{x}_{2}^{*}=0$ (Zhao e Thornton, 1992). Utilizando este fato e a definição de ponto de equilíbrio, obtém-se:

$$
P=\left(x_{1}^{*}=\frac{u_{M}}{R}, \quad x_{2}^{*}=0, \quad x_{3}^{*}=\sqrt{\frac{c}{2 m g}} \frac{u_{M}}{R}\right)
$$

\subsection{Linearização em Torno do Ponto de Equilíbrio}

Para pequenos desvios do estado estacionário do sistema Maglev, como mostrado na Fig. 2, pode-se escrever:

$$
s_{M}=S_{0}+s, \quad I_{M}=I_{0}+I, \quad u_{M}=U_{0}+u, \quad \dot{S}_{0}=0, \quad \dot{I}_{0}=0
$$


Por outro lado, na posição de equilíbrio do veiculo, tem-se $L_{0}=L\left(S_{0}\right), U=I_{0} R$. Isolando $c$ na equação (1), tem-se $c=L\left(s_{M}\right) s_{M}$, no entanto, a partir das relações acima, para a posição de equilíbrio do sistema tem-se $s=0$, portanto $s_{M}=S_{0}, \log \mathrm{o} c=L\left(S_{0}\right) S_{0}$, ou ainda, $c=L_{0} S_{0}$ (Shu e Meisinger, 2011).

Uma forma de analisar a estabilidade de um sistema não linear é realizar a linearização em torno do seu ponto de equilíbrio (Monteiro, 2011). Assim, a partir do sistema não linear (5), tem-se que, as funções $\dot{x}_{1}=f\left(x_{1}, x_{2}, x_{3}\right)$, $\dot{x}_{2}=g\left(x_{1}, x_{2}, x_{3}\right)$ e $\dot{x}_{3}=h\left(x_{1}, x_{2}, x_{3}\right)$ em torno de $P=\left(x_{1}^{*}, x_{2}^{*}, x_{3}^{*}\right)$ dado por (6), podem ser aproximadas por equações de reta. Para isso, expandem-se essas funções em série de Taylor, o que resulta na seguinte matriz:

$$
\mathcal{J}=\left[\begin{array}{lll}
\partial f / \partial x_{1} & \partial f / \partial x_{2} & \partial f / \partial x_{3} \\
\partial g / \partial x_{1} & \partial g / \partial x_{2} & \partial g / \partial x_{3} \\
\partial h / \partial x_{1} & \partial h / \partial x_{2} & \partial h / \partial x_{3}
\end{array}\right]_{P} \Rightarrow \mathcal{J}=\left[\begin{array}{ccc}
\frac{-u_{M}}{L_{0} S_{0}} \sqrt{\frac{L_{0} S_{0}}{2 m g}} & \sqrt{\frac{2 m g}{L_{0} S_{0}}} & 0 \\
\frac{-2 R g}{u_{M}} & 0 & \frac{L_{0} S_{0} R}{m u_{M}}\left(\frac{2 m g}{L_{0} S_{0}}\right)^{3 / 2} \\
0 & 1 & 0
\end{array}\right]_{P}
$$

Em que, a partir da relação $c=L_{0} S_{0}$ e $P$ dado por (6), obtém-se a matriz jacobiana (7) para o ponto de equilíbrio do sistema (5).

\subsection{Classificação do Ponto de Equilíbrio e Simulação Computacional}

A estabilidade do ponto de equilíbrio (6) do sistema (5) pode ser analisada a partir do calculo dos autovalores da matriz jacobiana (7). Então a partir $\operatorname{de} \operatorname{det}(\mathcal{J}-\mathcal{I} \lambda)=0$, obtém-se o seguinte polinômio característico:

$$
a_{0} \lambda^{3}+a_{1} \lambda^{2}+a_{2} \lambda+a_{3}
$$

sendo, os coeficientes $a_{j}$ dados por:

$$
a_{0}=-1 \quad a_{1}=-\frac{u_{M}}{L_{0} S_{0}} \sqrt{\frac{L_{0} S_{0}}{2 m g}} \quad a_{2}=\left[\frac{L_{0} S_{0} R}{m u_{M}}\left(\frac{2 m g}{L_{0} S_{0}}\right)^{3 / 2}-\frac{2 R g}{u_{M}} \sqrt{\frac{2 m g}{L_{0} S_{0}}}\right] \quad a_{3}=\frac{1}{m}\left[\sqrt{\frac{L_{0} S_{0}}{2 m g}}\left(\frac{2 m g}{L_{0} S_{0}}\right)^{3 / 2}\right]
$$

Os autovalores da matriz jacobiana (7) são obtidos a partir do calculo das raízes do polinômio característico (8). Para tal, os seguintes parâmetros são utilizados.

Tabela 1. Parâmetros do modelo Maglev

\begin{tabular}{cccccc}
\hline$m[k g]$ & $g\left[m / s^{2}\right]$ & $S_{0}[m]$ & $L_{0}[h]$ & $R[\Omega]$ & $u_{M}[v]$ \\
\hline 10000 & 9.8 & 0.01 & 0.1 & 1 & 140 \\
\hline
\end{tabular}

Tais parâmetros podem ser encontrados em (Zhao e Thornton, 1992). Os autovalores obtidos, são:

$$
\lambda_{1}=-17.0036-22.9629 \mathrm{i}, \lambda_{2}=-17.0036+22.9629 \mathrm{i}, \lambda_{3}=24.0072
$$

Portanto, a partir dos autovalores calculado acima, tem-se que o ponto de equilíbrio $P=(140,0,0.01)$ calculado a partir de (6) com o uso dos parâmetros da Tab. 1 é um nó sela, o qual é instável no sentido de Lyapunov.

A Figura 3 mostra o retrato de fases na projeção $x_{2}-x_{3}$. Os parâmetros de simulação são os valores apresentados na Tab. 1 e as equações de movimento foram integradas numericamente com o MATLAB ${ }^{\circledR}$ a partir de varias condições iniciais na vizinhança do ponto de equilíbrio do sistema.

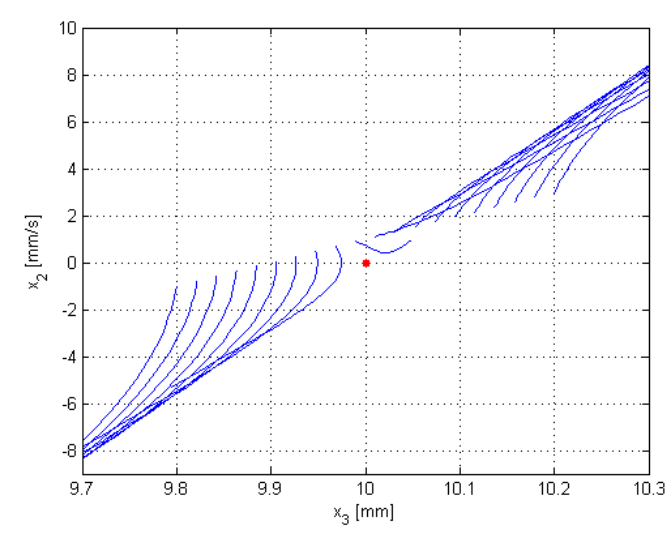

Figura 3. Retrato de fases da projeção $x_{2}-x_{3}$. 
Note que, pelo Teorema das Variedades Hiperbólicas há $n_{e}$ autovalores com parte real negativa (subespaço estável $\left.E^{e}\right), n_{i}$ autovalores com parte real positiva (subespaço instável $E^{i}$ ) e $n_{c}$ autovalores com parte real nula (subespaço central $E^{c}$ ), então $n=n_{e}+n_{i}+n_{c}$, sendo $n$ a dimensão do sistema. Como, não há nenhum autovalor com parte real nula, ou seja, $\operatorname{Re}(\lambda) \neq 0$, então $n_{c}=0$, assim o ponto de equilíbrio é hiperbólico, e o Teorema de Hartman-Grobman é válido, ou seja, a estabilidade do ponto de equilíbrio hiperbólico é preservada quando se lineariza o sistema em torno deste ponto (Monteiro, 2011).

Por fim, a partir dos autovalores calculados tem-se o seguinte: $\lambda_{1,2}=-17.0036 \pm 22.9629 i$, cujos autovetores localizam-se no plano $x_{1}$ x $x_{2}$, que é portanto, o subespaço estável $E^{e}$; e $\lambda_{3}=24.0072$, cujo autovetor coincide com $x_{3}$, que constitui o subespaço instável $E^{i}$. Consequentemente, existe uma variedade bidimensional (uma solução do sistema não linear original) que é tangente a $E^{e}$ no ponto de equilíbrio $P$ e se aproxima desse ponto para $t \rightarrow \infty$; assim como existe uma variedade unidimensional que é tangente a $E^{i}$ no ponto de equilíbrio $P$ e se afasta desse ponto para $t \rightarrow \infty$. A variedade estável divide o espaço de estados em duas partes: trajetórias na parte superior próximo a $x_{3} \rightarrow \infty$ ao longo de trajetórias instáveis, correspondendo ao caso em que o veículo cai do trilho; e trajetórias na parte inferior próximo ao plano $x_{3}=0$ ao longo de trajetórias instáveis, correspondendo ao caso em que o veículo colide com o trilho (Zhao e Thornton, 1992).

\section{CONTROLE UTILIZANDO A EQUAÇÃO DE RICCATI DEPENDENTE DO ESTADO (SDRE)}

Várias técnicas para síntese de controladores para sistemas não lineares foram desenvolvidas, algumas destas são: Linearização por jacobiano ("Feedback Linearization"); Controle Adaptativo; "Gain Sheduling"; Controle por Modo Deslizante, etc. Contudo, a lei de controle geralmente é determinada por algum método de análise, como os métodos baseados em funções de Lyapunov, logo, estes métodos resultam em controladores pouco intuitivos para a implementação prática. Erdem e Alleyne (2004) argumentam que, até a publicação do trabalho "Nonlinear Regulation and Nonlinear $H_{\infty}$ Control Via the State-Dependent Riccati Equation Techniques" desenvolvido em 1996 por James R. Cloutier e outros autores, não se dispunha de um bom método de projeto que permitisse estabelecer um compromisso entre o erro de estado e o esforço de controle para sistemas não lineares, como o método LQR (Carvalho, 2005).

Segundo Carvalho (2005), Cloutier em 1996, dentro do espírito do regulador LQR, aplicou a equação de Riccati a sistemas não lineares desenvolvendo a metodologia da Equação de Riccati Dependente do Estado (State Dependent Riccati Equation - SDRE). O nome SDRE vem da construção da lei de controle, e de forma resumida o que se faz é: primeiramente um sistema linear (também chamado de forma pseudo-linear) dependente do estado é obtido do modelo não linear; os parâmetros de controle são determinados pela solução da Equação de Riccati ponto a ponto no espaço de estado. A lei de controle é, em geral, uma solução sub-ótima de horizonte infinito.

\subsection{Algoritmo do Controle $S D R E$}

A técnica de controle SDRE para obter a solução ótima para o problema dinâmico de controle, tem o seguinte procedimento (Shawky et al., 2007):

Passo 1: Definir o espaço de estado do modelo e parametrizar o modelo na forma de coeficientes dependente do estado (SDC);

Passo 2: Mensurar o estado do sistema $x(t)$, ou seja, definir $x(0)=x_{0}$, e escolher os coeficientes das matrizes $Q$ e $R$;

Passo 3: Resolver a equação de Riccati dependente dos estados dada por:

$$
A^{T}(x) P(x)+P(x) A(x)-P(x) B(x) R^{-1}(x) B^{T}(x) P(x)+Q(x)=0
$$

Passo 4: Calcular o sinal de entrada, dado por:

$$
u=-R^{-1}(x) B^{T}(x) P(x) x
$$

Passo 5: Assumir o valor de saída do sistema $\dot{x}=A(x) x+B(x) u ; y=S(x) x$ como um novo valor inicial e atualizar o estado do sistema $x(t)$. Voltar ao passo 3 e repetir o processo até que o critério de parada definido seja alcançado.

\subsection{Parametrização}

Um sistema não linear definido por $\dot{x}=f(x)+g(x)$ pode ser parametrizado em uma equação diferencial de primeira ordem e representado pela seguinte estrutura pseudo-linear:

$$
\begin{aligned}
& \dot{x}=A(x) x+B(x) u \\
& y=C(x) x
\end{aligned}
$$

onde $f(x)=A(x) x$ e $g(x)=B(x)$, sendo $A(x)$ e $B(x)$ dependentes do estado; $A(x) \in \Re^{n x n}$ é a matriz dinâmica; $B(x) \in \Re^{n x m}$ é a matriz de entrada; $C$ é a matriz de saída do sistema; $x \in \Re^{n}$ é o vetor de estados do sistema; $u \in U \in \Re^{m}$ é a função de controle, $U$ é a força aplicada no controle; $y \in \Re^{s}$ ( $\Re^{s}$ é a dimensão do vetor de saída do sistema) (Chavarette, 2013). 
É importante ressaltar que a forma pseudo-linear dependente do estado (11) pode ser apresentada de vários modos a partir de um sistema não linear, logo a escolha de $A(x)$ não é única, ou seja, em geral há infinitas parametrizações que correspondem a uma mesma função $f(x)$ resultando em diferentes matrizes $A(x)$ (Chavarette, 2013).

Associadas à forma pseudo-linear com coeficientes dependentes do estado $(S D C)$, têm-se as seguintes definições (Chavarette, 2013):

Definição 1. $A(x)$ é uma parametrização observável (detectável) do sistema não linear [na região $\Omega$ ] se o par $(C(x), A(x))$ é ponto a ponto observável (detectável) no sentido linear para todo $x[\in \Omega]$.

Definição 2. A(x) é uma parametrização controlável (estabilizável) do sistema não linear [na região $\Omega$ ] se o par $(A(x), B(x))$ é ponto a ponto controlável (estabilizável) no sentido linear para todo $x[\in \Omega]$.

O método SDRE requer que o vetor de estados completo esteja disponível e o par $(A(x), B(x))$ seja controlável ponto a ponto no contexto de sistema lineares $\forall x$. Assim, um fator importante é que a escolha das parametrizações não viole a controlabilidade, a qual pode ser verificada através da matriz de controlabilidade como no caso de sistemas lineares, verificando-se se a referida matriz tem posto completo no domínio de interesse. Esta condição simplesmente garante que a equação algébrica de Riccati tenha solução para um estado particular $x$ nesse domínio. A controlabilidade ponto a ponto não necessariamente equivale à controlabilidade não linear. Devido à não unicidade de $A(x)$, diferentes escolhas de $A(x)$ podem produzir diversas matrizes de controlabilidade e, portanto, distintas características de controlabilidade ponto a ponto (Carvalho, 2005).

Lema 1. Para existir uma única solução ponto a ponto para a equação de Riccati, é suficiente que o sistema (11) seja estabilizável e detectável ponto a ponto.

Considerando as condições iniciais e finais como: $x\left(t_{0}\right)=x_{0}, x(\infty)=0$, os coeficientes dependentes do estado $(S D C)$ são dados por:

$$
\begin{gathered}
A(x)=\left[\begin{array}{ccc}
\frac{R x_{3}}{L_{0} S_{0}}+\frac{x_{2}}{x_{3}} & \frac{x_{1}}{x_{3}} & \frac{u_{M}-2 R x_{1}}{L_{0} S_{0}}-\frac{x_{1} x_{2}}{x_{3}^{2}} \\
-\frac{L_{0} S_{0} x_{1}}{m x_{3}^{2}} & 0 & \frac{L_{0} S_{0} x_{1}^{2}}{2 m x_{3}^{3}} \\
0 & 1 & 0
\end{array}\right] \\
Q(x)=S^{T}(x) S(x) \quad \text { sendo, }
\end{gathered}
$$

Portanto, o sistema (5) representado na forma (11) é:

$$
\dot{\mathbf{x}}=\left[\begin{array}{ccc}
\frac{x_{2}}{x_{3}}+1000 x_{3} & \frac{x_{1}}{x_{3}} & -\frac{x_{1} x_{2}}{x_{3}^{2}}-2000 x_{1}+140000 \\
-\frac{10^{-7} x_{1}}{x_{3}^{2}} & 0 & \frac{5 \times 10^{-8} x_{1}^{2}}{x_{3}^{3}} \\
0 & 1 & 0
\end{array}\right] \mathbf{x}+\left[\begin{array}{l}
1 \\
0 \\
0
\end{array}\right] u
$$

O estado x e o controle $u$ são dados pela função $f(x)=A(x) x, g(x)=B(x)$ e $C(x)=S(x)$.

\subsection{Aplicação do Método SDRE para o Projeto do Controlador e Simulações Computacionais}

O funcional de custo (índice de desempenho) a ser minimizado através do controle SDRE, é dado por:

$$
J=\frac{1}{2} \int_{t_{0}}^{\infty}\left[x^{T} Q(x) x+u^{T} R(x) u\right] d t
$$

sendo $Q(x)$ uma matriz definida positiva ou semi-definida positiva e $R(x)$ definida positiva; as quais determinam a importância relativa do estado $x$ e o consumo de energia $u$ respectivamente (Chavarette, 2013).

Os coeficientes escolhidos para as matrizes $Q$ e $R$, são:

$$
Q=\left[\begin{array}{ccc}
1 & 0 & 0 \\
0 & 1 & 0 \\
0 & 0 & 6.31 \times 10^{7}
\end{array}\right] \quad R=\left[8 \times 10^{-5}\right]
$$

O controle por realimentação de estados utilizando o controle $S D R E$ é dado por (10) e a matriz $P(x)$ é a equação de Riccati dependente dos estados dada por (9).

Segundo Mracek e Cloutier citado por Chavarette (2013), na região $\Omega$ sobre a origem o método SDRE garante uma solução assintoticamente estável em malha fechada. No caso de um escalar, o método $S D R E$ atinge a solução ótima para o funcional (15), mesmo quando $Q$ e $R$ são funções de $x$.

As Figuras 4(a), 4(b) e 4(c) mostram a evolução temporal das variáveis de estado $x_{1}, x_{2}$ e $x_{3}$ respectivamente, para o sistema sem controle (trajetória em azul) e com controle (trajetória em preto). A Figura 4(d) mostra uma trajetória do sistema controlado no espaço de estados. Os parâmetros de simulação são os valores apresentados na Tab. 1 e as equações de movimento foram integradas numericamente com o MATLAB ${ }^{\circledR}$. 


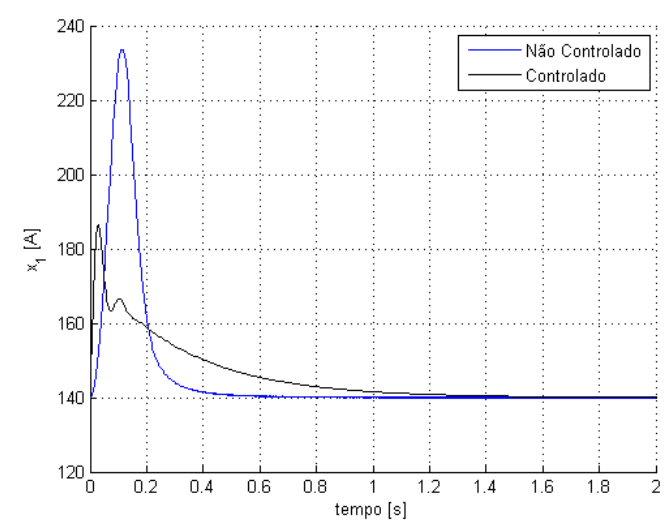

(a) Hístórico no tempo de $x_{1}$.

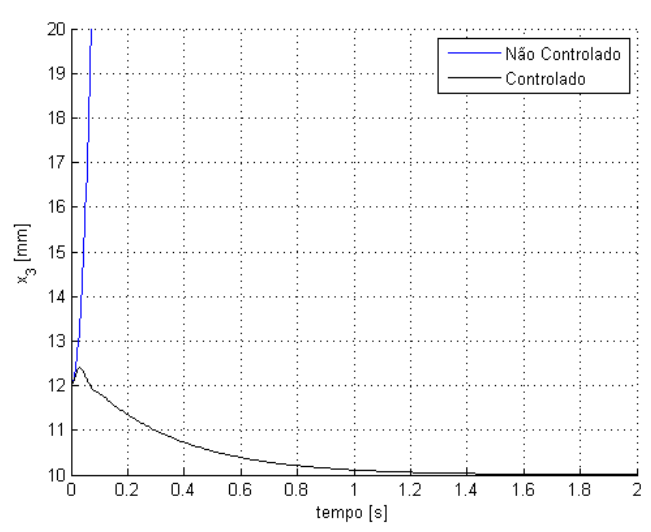

(c) Hístórico no tempo de $x_{3}$.

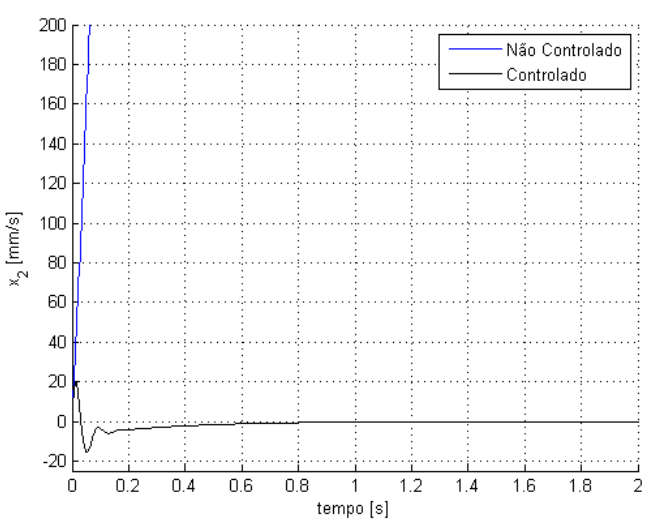

(b) Hístórico no tempo de $x_{2}$

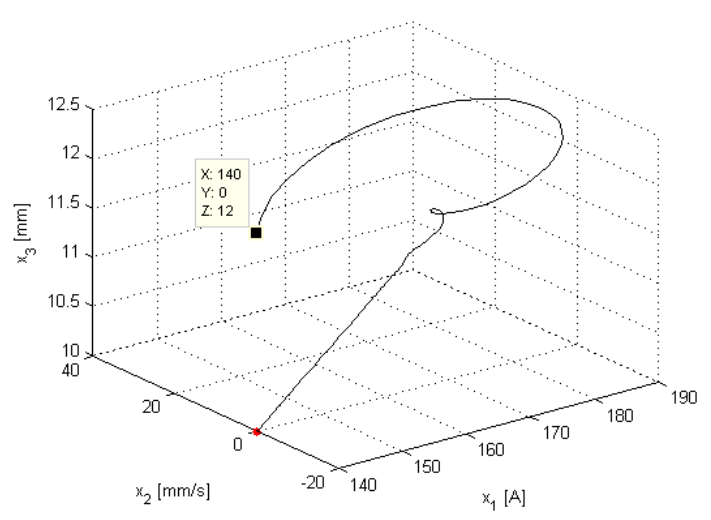

(d) Trajetória do sistema controlado no espaço de estados.

Figura 4. Evolução temporal das variáveis de estado $\left(\mathrm{x}_{1}, \mathrm{x}_{2}, \mathrm{x}_{3}\right)$ e trajetória do sistema controlado no espaço de estados; $x(0)=(140,0,0.012)$.

As simulações do sistema controlado foram realizadas, considerando que as variáveis de estado permaneçam no seguinte domínio $\left\{\left(x_{1}, x_{2}, x_{3}\right) \in \Re \quad \mid \quad 0 \leq x_{1} \leq 200[A],-100 \leq x_{2} \leq 100[\mathrm{~mm} / \mathrm{s}], 0<x_{3} \leq 15[\mathrm{~mm}]\right\}$. Assim, as trajetórias nas figuras acima foram obtidas por integração numérica, considerando um desvio inicial de $\mathbf{2 m m}$, da posição de equilíbrio do sistema, o qual está representado pelo ponto vermelho (Fig. 4(d)). Note que, o controlador proposto foi capaz de estabilizar o sistema com um desvio máximo da posição de equilíbrio menor do que 2.5mm, como pode ser visto na Fig. 4(c). Para um desvio inicial da posição de equilíbrio maior do que $\mathbf{2 m m}$ a variável de estado $x_{1}>200 A$, no entanto, a corrente não pode elevar-se o suficiente (pois $x_{1} \leq 200 A$ ) para trazer o sistema para a posição de equilíbrio.

\section{CONCLUSÕES}

Um sistema de levitação eletromagnética, tal como o Maglev Transrapid foi simplificado por um sistema de massa única sobre um trilho rígido e sem a suspensão secundária, a fim de estudar sua estabilidade. O modelo possui um estado de equilíbrio a partir do instante em que a força magnética provida dos eletroímãs contrabalanceia exatamente a força gravitacional (peso) do veículo, e assim o mesmo tem velocidade e aceleração vertical nula, ou seja, $x_{2}=\dot{x}_{2}=0$, entretanto, o equilíbrio é um nó sela, o qual é instável no sentido de Lyapunov. Deste modo, tornou-se necessário projetar algum controlador para o sistema, a fim de que o mesmo seja estabilizado e mantenha-se a uma distância constante do trilho. Assim, foi proposto um controle utilizando a equação de Riccati dependente do estado (SDRE), o qual demonstrou ser eficiente para o problema abordado neste trabalho; de modo que o modelo com suspensão eletromagnética estudado pode ser estabilizado utilizando está técnica de controle. Uma sugestão de trabalho futuro é a seguinte: na prática, a velocidade vertical do veículo não pode ser mensurada por um sensor e a medição da aceleração vertical e da distância entre o veículo e o trilho são influenciadas por ruídos, assim um observador tal como o KALMAN-FILTER pode ser implementado para estimar estes estados.

\section{AGRADECIMENTOS}

Os autores agradecem ao CNPQ (Proc. $n^{\circ}$ 132786/2013-3, Proc. $\mathrm{n}^{\circ}$ 301769/2012-5) pelo apoio financeiro da pesquisa. 


\title{
REFERÊNCIAS
}

Carvalho, L. K. 2005. Sobre a estabilização global de sistemas não lineares via Equação de Riccati Dependente do Estado. Dissertação de Mestrado, Universidade Federal do Rio de Janeiro - UFRJ.

URL http: / / www.pee.ufrj.br/teses / ?Resumo=2005112501

Chavarette, F. R. 2013. Control design applied to a non-ideal structural system with behavior chaotic. International Journal of Pure and Applied Mathematics, 86, 487-500.

URL http: / / www. i jpam. eu/contents/2013-86-3/3/

David, E. 2009. O futuro das estradas de ferro no Brasil. Portifolium, 1 ed.

Erdem, E. B., e Alleyne, A. G. 2004. Design of a class of nonlinear controller via state dependent riccati equations. IEEE Transactions on Control Systems Technology, 12, 133-137.

URL http: / / ieeexplore. ieee.org/xpls/abs_all.jsp?arnumber=1268058\&tag=1

Lee, C.-Y., Jo, J.-M., Lee, J.-H., Kim, I.-H., e Han, Y.-J. 2013. Development of superconducting-hybrid magnetic levitation system for high-speed ems maglev. IEEE International Conference on Electrial Machines and Systems, (pp. 1922-1927).

URL http: / / ieeexplore.ieee.org/xpls/abs_all.jsp?arnumber=6713231

Lemos, N. A. 2007. Mecânica Analítica. Livraria da Física, 2 ed.

Monteiro, L. H. A. 2011. Sistemas Dinâmicos. Livraria da Física, 3 ed.

Motta, E. S. 2011. Otimização de trinho magnético de um sistema de levitação supercondutora para veículo Maglev. Tese de Doutorado, Instituto Alberto Luiz Coimbra de Pós-Graduação e Pesquisa de Engenharia - Universidade Federal do Rio de Janeiro - UFRJ.

URL http: / / www.pee.ufrj.br/teses/textocompleto/2011110301.pdf

Shawky, A. M., Ordys, A. W., Petropoulakis, L., e Grimble, M. J. 2007. Position control of flexible manipulator using non-linear h with state-dependent riccati equation. Journal Of Systems And Control Engineering, (pp. 475-486).

URL http://pii.sagepub.com/content/221/3/475. short

Shu, G., e Meisinger, R. 2011. State estimation and simulation of the magnetic levitation system of a high-speed maglev train. 2011 International Conference on Electronic \& Mechanical Engineering and Information Technology, (pp. 944947).

URL http: / / ieeexplore. ieee.org/xpls/icp.jsp?arnumber $=6023250$

Zhao, F., e Thornton, R. 1992. Automatic design of a maglev controller in state space. (pp. 2562-2567).

URL http: / / ieeexplore. ieee.org/stamp/stamp.jsp?tp=\&arnumber $=371062$

\section{RESPONSABILIDADE AUTORAL}

“Os autores são os únicos responsáveis pelo conteúdo deste trabalho".

\section{STABILITY ANALYSIS AND CONTROL DESIGN BASED ON SDRE METHOD FOR A MAGLEV SYSTEM SIMPLIFIED}

\author{
Thalles Denner Ferreira Cabral, ctdf. engmecegmail.com ${ }^{1}$ \\ Fábio Roberto Chavarette, fabioch@mat . feis . unesp.br ${ }^{1}$ \\ ${ }^{1}$ UNESP - Universidade Estadual Paulista - Departamento de Engenharia Mecânica \\ Avenida Brasil, 56, 15385-000, Ilha Solteira, SP, Brasil
}

\begin{abstract}
Maglev trains (Magnetic levitation transport) are transport systems that can reach high speeds with low friction compared to conventional wheel-rail trains. Other factors that become the technology very attractive Maglev trains is that it can reduce the traffic congestion and reduce environmental impacts additionally. Several engineers around the world, have been developing some projects related with the technology Maglev trains, being that some these projects are in a advanced stage relatively, for example, the experimental Maglev systems of Germany and Japan, which have demonstrated that this mode of transportation can compete with air transport profitably. The first train system that operates completely without contact and wheels was developed by the Germans, which have been constructed with electromagnetic suspension (EML). This paper researches the stability of a system with electromagnetic levitation (EML) such as the German Transrapid Maglev, from a simplified model with a single mass on a rigid rail and without secondary suspension. The motion equations were obtained by the Lagrangian formulation of classical mechanics. Because of the instability of the electromagnetic levitation system, the electromagnet magnetic force must be controlled actively; therefore we used the method SDRE (State Dependent Riccati Equation) for the controller design. For the simulation of the control system MATLAB ${ }^{\circledR}$ software is used, in order to show the evolution of the state variables in time and the trajectory of the controlled system in the state space.
\end{abstract}

Keywords: computer simulation, electromagnetic levitation, mathematical model, SDRE control, stability 